\title{
PROGNOSTIC POWER OF INFLAMMATORY RESPONSE SCORING SYSTEMS IN PERIAMPULLARY PANCREATIC CANCER: A SYSTEMATIC REVIEW
}

\author{
Majid Ali, Alexia Farrugia, Ricky Bhogal, Saboor Khan, Gabriele Marangoni, Jawad Ahmad \\ Department of Upper Gastrointestinal and Hepatobiliary Surgery, University Hospitals of Coventry and \\ Warwickshire, United Kingdom \\ Received: 21 September 2019 / Accepted: 10 June 2019
}

\begin{abstract}
Introduction: Assessment of systemic inflammatory response forms the basis of several scoring systems that attempt to prognosticate patients with periampullary pancreatic carcinoma (PPC). We assessed the validity of three of these scoring systems for patients' prognosis following intervention for PPC: Glasgow prognostic score (GPS) and its modified version (mGPS), platelet-lymphocyte ratio (PLR) and neutrophil-lymphocyte ratio (NLR).
\end{abstract}

Methods: EMBASE and MEDLINE databases were searched for all published studies until September 2018 using comprehensive text word and MeSH terms. Meta-analysis of observational studies in epidemiology guidelines was followed. All identified studies were analysed and relevant studies were included in the review.

Results: Three studies which assessed the role of GPS, four studies that evaluated the use of NLR and three that assessed the role of PLR in patients with PPC were identified. None of these studies demonstrated any value in the pre-operative assessment of patients with PPC. The limited number of studies available precluded further statistical analysis.

Conclusions: Based on available evidence, GPS, NLR and PLR do not appear to be useful scoring systems to predict prognosis of patients with PPC. Larger studies are warranted before the application of inflammatory scoring systems could be recommended in patients with PPC.

Key words: Periampullary cancer, Glasgow prognostic score, modified Glasgow prognostic score, plateletlymphocyte ratio, neutrophil-lymphocyte ratio

\section{Introduction}

Periampullary pancreatic cancer (PPC) has an overall 5-year survival varying from 36 to $51 \%$, with a median survival of 66 months in patients who undergo resection. ${ }^{[1-3]}$ Surgical resection is the only curative treatment for such cancers; however, resection is only possible in $15-20 \%$ of patients. $^{[3]}$ The mainstay of surgical resection is in the form of either pylorus-preserving pancreaticoduodenectomy (PPPD) or Kausch-Whipple's procedure. Both PPPD and KauschWhipple's procedure have a reported perioperative morbidity of approximately $40 \%{ }^{[4]}$ and a reported mortality of $3 \%$ in high volume specialised centres. ${ }^{[5]}$

Correspondence: Dr. Majid Ali, Department of Upper

Gastrointestinal and Hepatobiliary Surgery, University Hospitals of

Coventry and Warwickshire, United Kingdom.

Email: majidandfriends@gmail.com
Improved patient selection, better imaging techniques and the introduction of adjuvant chemotherapy have improved patients' outcomes in recent years. However, tumour behaviour remains difficult to assess preoperatively with only detailed post-operative histological examination allowing meaningful prognostic factors, such as lymph node status, histological subtype, tumour stage and margin status, to be fully defined.

There is thus a need for robust markers or biomarkers that could be used in the prognostic stratification of patients with PPC before surgical resection.

Scoring systems based on the assessment of the systemic inflammatory response have been shown to be useful preintervention indicators of prognosis in various neoplastic conditions. ${ }^{[6-8]}$ Commonly used scoring systems are the 
Glasgow prognostic score (GPS), including its modified version (mGPS), the platelet-lymphocyte ratio (PLR) and the neutrophil-lymphocyte ratio (NLR). However, none of them have been adopted routinely into clinical practice and few have been externally validated. The role of these scoring systems in patients with PPC remains unexplored.

The aim of this review was to evaluate the evidence for the prognostic value of these scoring systems in predicting outcomes in patients with PPC, specifically assessing the ability to predict patient survival.

\section{Materials and Methods}

A literature search was performed to identify all studies that examined the prognostic value of one or more of the three scoring systems in patients with PPC. All studies published from 1966 to $1^{\text {st }}$ week September 2018 were evaluated. EMBASE and MEDLINE databases were searched using comprehensive text and $\mathrm{MeSH}$ terms. Keywords used for search are listed as under (periampullary cancer*, periampullary carcinoma*, periampullary tumours*, periampullary adenocarcinoma*, GPS*, modified GPS*, GPS* or mGPS*, PLR*, PLR*, NLR* and NLR*). Google Scholar was used to identify additional manuscripts. A bibliography of included articles was hand searched for relevant additions. Two investigators independently searched and analysed all data, and consensus was sought with a third author where discrepancies existed.

Since all studies were observational, our search protocol was based on meta-analysis of observational studies in epidemiology guidelines. ${ }^{[9]}$ All papers indexed in the English language were included. Case reports or case series, unpublished abstracts, proceedings of meetings and animal studies were excluded. After removal of duplicates and irrelevant articles, abstracts were screened for further exclusions. Full-text articles were then read before inclusion in the analysis. Assessment of bias in the studies was made using the risk of bias in nonrandomised studies of interventions (ROBINS-I) tool by two reviewers independently. The ROBINS-I tool assesses bias across six domains including confounding, participant selection, intervention classification, departure from intended interventions, missing data, measurement of outcomes and selection of reported results. For each domain, an outcome of low, moderate, serious, critical and no information for risk of bias is recorded. An overall risk of bias judgement is then determined through combination of the six domains.

The primary aim of our review was to assess if any of these scoring systems could be used to predict patient outcome in PPC.

\section{Results}

The results from searching the EMBASE and MEDLINE databases are presented in Table 1. Among the included studies, one focused on the prognostic value of GPS,

\section{Table 1: Search strategy (no filters) and results from searching EMBASE and MEDLINE databases}

\begin{tabular}{|c|c|c|}
\hline Search strategy & MEDLINE $^{Y}$ & EMBASE $^{\phi}$ \\
\hline $\begin{array}{l}\text { Periampullary cancer/ } \\
\text { carcinoma/tumour/ade- } \\
\text { nocarcinoma }\end{array}$ & 786 & 1265 \\
\hline $\begin{array}{l}\text { Glasgow prognostic } \\
\text { score* }\end{array}$ & 523 & 843 \\
\hline $\begin{array}{l}\text { Modified Glasgow prog- } \\
\text { nostic score* }\end{array}$ & 217 & 368 \\
\hline GPS* or mGPS* & 22350 & 30622 \\
\hline 2 or 3 or 4 & 22440 & 30740 \\
\hline $\begin{array}{l}\text { Prognostic nutrition } \\
\text { index* }\end{array}$ & 43 & 70 \\
\hline $\begin{array}{l}\text { Onodera's prognostic } \\
\text { nutrition index* }\end{array}$ & 3 & 6 \\
\hline PNI* & 1332 & 2254 \\
\hline 6 or 7 or 8 & 1352 & 2276 \\
\hline PLR* & 427 & 1713 \\
\hline PLR* & 2000 & 3090 \\
\hline 10 or 11 & 2083 & 3505 \\
\hline NLR* & 1424 & 5552 \\
\hline NLR* & 7122 & 7265 \\
\hline 13 or 14 & 7553 & 9033 \\
\hline 5 or 9 or 12 or 15 & 31982 & 43026 \\
\hline 1 and 16 & 3 & 8 \\
\hline 1 and 5 & 0 & 5 \\
\hline 1 and 9 & 1 & 3 \\
\hline 1 and 12 & 1 & 2 \\
\hline 1 and 15 & 1 & 2 \\
\hline
\end{tabular}


two assessed NLR and three investigated the value of PLR in PC.

Three studies evaluated GPS in patients with PPC periods. $^{[10,11]}$ Garcea et al. described 137 patients with potentially resectable PPC lesions detected on preoperative imaging who were assessed for resectability at laparoscopy. Five patients had PPC in this series. Patient outcome after surgical intervention was not reported. GPS was assessed to see if it could be used to discriminate operability before laparoscopy, but no statistical difference was found. ${ }^{[10]}$ Lowe et al. assessed that 302 patients had pancreatic resection for periampullary cancers, of which 45 had histologically confirmed PPC and 96\% had an R0 resection. The data presented in this study did not allow for calculation of the GPS, and hence, this could not be assessed in the presented review. Kumamoto et al. assessed 84 patients and found that the GPS did not accurately predict postoperative major complications. ${ }^{[12]}$

Four studies evaluated NLR in patients with PPC. ${ }^{[10,12-14]}$ Garcea et al. demonstrated that NLR has no pre-operative value in discriminating between operable and non-operable disease and has no value in detecting occult disease. ${ }^{[10]}$ Kumamoto et al. found that a higher NLR as associated with major complications. ${ }^{[12]}$ Hamed et al. evaluated the use of NLR in 74 patients with periampullary tumours; they demonstrated that the median survival between patients having NLR $>5$ and $<5$ was not significantly different; however, in patients developing recurrence, NLR $<5$ was associated with improved survival. ${ }^{[13]}$ Demirci et al. found that an NLR of more than 3 was associated with overall survival and disease-free survival. ${ }^{[14]}$

Three studies aimed to assess the role of PLR in patients with PC. ${ }^{[10,15-17]}$ Garcea et al. showed no role of PLR in assessing the benefit of staging laparoscopy in PPC patients. Smith et al. included 263 pancreatic resections in their study, of which 48 had PPC. The authors demonstrated that pre-operative median PLR pf $<$ or $=150$ was significantly associated with locally advanced and metastatic disease in patients with periampullary lesions $(P<0.001)$, but this group included pancreatic ductal adenocarcinoma, cholangiocarcinoma and metastatic adenocarcinoma. Overall patient survival was not presented in this study although median PLR was specifically associated with increased risk of nodal disease in patients with PC $(P=0.002)$. Demirci et al. assessed 82 patients with PPC and found that a PLR $>212$ was associated with a significantly worse overall survival. ${ }^{[17]}$

The limited data and heterogeneity of the studies were such that a meta-analysis was not possible and only a descriptive analysis of the studies was made.

\section{Discussion}

Patient outcome following surgical intervention for PPC depends on both tumour biology and patientrelated factors. The systemic inflammatory response is acknowledged to be an important factor in many tumours including PPC. ${ }^{[18]}$ The mechanism of PPC development remains incompletely understood and is likely to involve chronic inflammation, immune cell activation and growthpromoting signalling pathways. ${ }^{[19]}$ The inflammatory response may serve as a potential parameter to help prognosis stratification with the aim to improve patient's outcome.

In this review, we assessed elements of the inflammatory response evaluating the GPS, NLR and PLR scoring systems to investigate whether they can be helpful tools in patients' stratification and, in particular, if they could predict resectability. ${ }^{[7,20]}$ These scoring systems may allow pre-operative identification of aggressive tumours, thus changing the treatment strategy (e.g., neoadjuvant treatments) and provide individualised care. If reliable, they could also serve as useful monitoring tools after successful resection.

Many cancers have been shown to incite a systemic inflammatory response as evidenced by an elevated C-reactive protein (CRP). Indeed, a recent study by Shirai et al. has shown that in patients with pancreatic ductal cancer that demonstrates a significant inflammatory response have a poorer prognosis and poorer survival. ${ }^{[21]}$ The fine mechanisms of these events remain elusive, but the release of pro-inflammatory cytokines such as tumour necrosis factor $\alpha$ and interleukin- 6 , modulation of endothelial cell function and angiogenesis seems to play a significant role. ${ }^{[22]}$ 
Table 3: Characteristics of patients included in the individual studies used in the meta-analysis

\begin{tabular}{l|c|c|l}
\hline Study & $\begin{array}{c}\text { Total number } \\
\text { of patients }\end{array}$ & $\begin{array}{c}\text { Number } \\
\text { of PPC }\end{array}$ & $\begin{array}{l}\text { Parameter(s) } \\
\text { studied }\end{array}$ \\
\hline Garcea et al. & 137 & 5 & GPS, NLR, PLR \\
\hline Lowe et al. & 302 & 45 & Nil \\
\hline Hamed et al. & 228 & 61 & NLR \\
\hline Sakka et al. & 34 & 3 & PLR \\
\hline Smith et al. & 263 & 204 & PLR \\
\hline
\end{tabular}

PPC: Periampullary pancreatic carcinoma, GPS: Glasgow prognostic score, NLR: Neutrophil-lymphocyte ratio, PLR: Platelet-lymphocyte ratio

GPS provides an objective scoring system that combines CRP and serum albumin. ${ }^{[23]}$ There are reports of adverse outcomes in patients with hepatobiliary malignancy and elevated GPS. ${ }^{[24]}$ Only one study was found which investigated the potential role of GPS in PPC, and unfortunately, this only assessed whether the scoring system could discriminate between patients with operable and non-operable disease. Therefore, it remains to be established if GPS or mGPS can be used as a pre-operative scoring system.

NLR is calculated from the differential count by dividing the absolute neutrophil count with the absolute lymphocyte count. NLR $>5$ is considered significant and a poor prognostic marker. ${ }^{[25]}$ However, no study has clearly assessed the role NLR in predicting patient survival following a diagnosis of PPC. Although one study in our review did suggest that patients with NLR $>5$ were associated with inferior patient outcome in periampullary tumours when PPC was assessed, NLR was found not to be predictive. ${ }^{[13]}$ It remains to be established if NLR could represent a useful tool in the pre-operative work-up in patients with PPC.

PLR is calculated in a similar manner as NLR but with the substitution of platelets for neutrophil count. There is some degree of discrepancy in defining a significant PLR; however, most studies stratified patients into three groups with a PLR $<150$, PLR $150-300$ and PLR $>300$ indicating good, moderate and poor prognosis, respectively. ${ }^{[26]}$ In our review, we found that two studies were from the same unit and covered the same study period. Hence, these data need to be interpreted carefully. The available literature does not allow for any firm 
conclusion as to the utility of PLR in patients with PPC. It is possible that PLR may be associated with poorer patient outcome, but it was not statistically significant.

It is important to note that the quality of the studies available for this meta-analysis was of low quality [Table 2] with many studies being retrospective and in some cases with low numbers of patient with PPC [Table 3].

\section{Conclusion}

There is a lack of evidence within literature regarding the prognostic use of inflammatory scoring systems in PPC. In addition, the available studies are of low quality with heterogeneous patient cohorts, low numbers and are retrospective in nature. Further research and larger studies are needed before recommending these scoring systems in patients with PPC.

\section{Conflict of Interest}

The authors declare that they have no conflict of interest.

\section{References}

1. Bourgouin S, Ewald J, Mancini J, et al. Predictors of survival in ampullary, bile duct and duodenal cancers following pancreaticoduodenectomy: A 10-year multicentre analysis. J Gastrointest Surg 2015;19:1247-55.

2. O'Connell JB, Maggard MA, Manunga J Jr., et al. Survival after resection of ampullary carcinoma: A national population-based study. Ann Surg Oncol 2008; $15: 1820-7$

3. Lemke J, Schäfer D, Sander S, et al. Survival and prognostic factors in pancreatic and ampullary cancer. Anticancer Res 2014;34:3011-20.

4. Poon RT, Fan ST. Decreasing the pancreatic leak rate after pancreaticoduodenectomy. Adv Surg 2008;42:33-48.

5. Shah OJ, Robbani I, Shah P, et al. A selective approach to the surgical management of periampullary cancer patients and its outcome. Hepatobiliary Pancreat Dis Int 2014;13:628-33.

6. McMillan DC. The systemic inflammation-based glasgow prognostic score: A decade of experience in patients with cancer. Cancer Treat Rev 2013;39:534-40.

7. Ahmad J, Grimes N, Farid S, et al. Inflammatory response related scoring systems in assessing the prognosis of patients with pancreatic ductal adenocarcinoma: A systematic review. Hepatobiliary Pancreat Dis Int 2014;13: 474-81.

8. Watt DG, Roxburgh CS, White M, et al. A survey of attitudes towards the clinical application of systemic inflammation based prognostic scores in cancer. Mediators Inflamm 2015;2015:7.

9. Stroup DF, Berlin JA, Morton SC, et al. Meta-analysis of observational studies in epidemiology: A proposal for reporting. Meta-analysis of observational studies in epidemiology (MOOSE) group. JAMA 2000;283:2008-12.

10. Garcea G, Cairns V, Berry DP, et al. Improving the diagnostic yield from staging laparoscopy for periampullary malignancies: The value of preoperative inflammatory markers and radiological tumor size. Pancreas 2012;41:233-7.

11. Lowe MC, Coban I, Adsay NV, et al. Important prognostic factors in adenocarcinoma of the ampulla of vater. Am Surg 2009;75:754-60.

12. Kumamoto Y, Kaizu T, Tajima H, et al. Neutrophil-tolymphocyte ratio as a predictor of postoperative morbidity in patients with distal cholangiocarcinoma. Mol Clin Oncol 2018;9:362-8.

13. Hamed MO, Roberts KJ, Smith AM, et al. Elevated pre-operative neutrophil to lymphocyte ratio predicts disease free survival following pancreatic resection for periampullary carcinomas. Pancreatology 2013; 13:534-8.

14. Demirci NS, Erdem GU. Prognostic role of neutrophil-tolymphocyte ratio (NLR) in patients with operable ampullary carcinoma. Bosn J Basic Med Sci 2018;18:268-74.

15. Sakka N, Smith RA, Whelan $\mathrm{P}$, et al. A preoperative prognostic score for resected pancreatic and periampullary neuroendocrine tumours. Pancreatology 2009;9: 670-6.

16. Smith RA, Bosonnet L, Ghaneh P, et al. The plateletlymphocyte ratio improves the predictive value of serum CA19-9 levels in determining patient selection for staging laparoscopy in suspected periampullary cancer. Surgery 2008;143:658-66.

17. Demirci NS, Ozdemir NY, Erdem GU, et al. Preoperative platelet-to-lymphocyte ratio is a predictor of prognosis in patients with ampullary carcinoma. Bratisl Lek Listy 2018;119:180-6.

18. McKay CJ, Glen P, McMillan DC. Chronic inflammation and pancreatic cancer. Best Pract Res Clin Gastroenterol 2008;22:65-73.

19. Ahn DH, Bekaii-Saab T. Ampullary cancer: An overview. Am Soc Clin Oncol Educ Book 2014;112-5.

20. Yamada S, Fujii T, Yabusaki N, et al. Clinical implication of inflammation-based prognostic score in pancreatic cancer: Glasgow prognostic score is the most reliable parameter. Medicine (Baltimore) 2016;95:e3582.

21. Shirai Y, Shiba H, Sakamoto T, et al. Preoperative platelet to lymphocyte ratio predicts outcome of patients with pancreatic ductal adenocarcinoma after pancreatic resection. Surgery 2015;158:360-5.

22. Momi N, Kaur S, Krishn SR, et al. Discovering the route from inflammation to pancreatic cancer. Minerva 
Gastroenterol Dietol 2012;58:283-97.

23. Forrest LM, McMillan DC, McArdle CS, et al. Comparison of an inflammation-based prognostic score (GPS) with performance status (ECOG) in patients receiving platinumbased chemotherapy for inoperable non-small-cell lung cancer. Br J Cancer 2004;90:1704-6.

24. Ni XC, Yi Y, Fu YP, et al. Prognostic value of the modified glasgow prognostic score in patients undergoing radical surgery for hepatocellular carcinoma. Medicine (Baltimore) 2015;94:e1486.

25. Gomez D, Farid S, Malik HZ, et al. Preoperative neutrophilto-lymphocyte ratio as a prognostic predictor after curative resection for hepatocellular carcinoma. World J Surg
2008;32:1757-62.

26. Templeton AJ, Ace O, McNamara MG, et al. Prognostic role of platelet to lymphocyte ratio in solid tumors: A systematic review and meta-analysis. Cancer Epidemiol Biomarkers Prev 2014;23:1204-12.

\section{Authorship Contributions}

Concept and design: MA, AF, RB, SK, GM, JA; Data collection and interpretation: $\mathrm{MA}, \mathrm{AF}, \mathrm{RB}$; Literature review and writing: MA, AF; Manuscript approval: MA, AF, RB, SK, GM JA 